\title{
Automatic Object Counting In Neubauer Chambers
}

\author{
Jayme Garcia Arnal Barbedo
}

\begin{abstract}
This paper presents a method to automate the object counting in Neubauer chambers. The proposed technique employs digital image processing to isolate the chamber grid markings and to identify each region of interest, which, in turn, enables the ability to perform the automatic counting for each region using the method that best suits the problem at hand. The technique's implementation includes an interface that allows the selection and combination of multiple regions according to the needs of the experiment. The capabilities of the method are illustrated by tackling the difficult problem of counting spores of the Clonostachys rosea fungus.
\end{abstract}

Keywords-Neubauer chamber, digital image processing, object counting, region of interest.

\section{INTRODUCTION}

Counting objects is one of the most basic activities in many different areas. This is particularly true in biological sciences, as counting microorganisms, cells, spores, among others, is arguably the most common and useful type of quantitative result.

Despite being such a widespread activity, the process of counting biological objects has experienced little automation so far. Even though efforts to automate some counting problems have been carried out [1]-[6], only in a few specific cases the process has become extensively automated (e.g. human blood cell counting [7]). In most cases, counting is still done by hand. However, even when performed manually, the process of counting objects is not always trivial or straightforward. In fact, most counting problems have peculiarities that make them tricky to tackle. For example, the objects may occur in very large numbers, and often appear clumped, making the counting very tiring and tedious, which in turn can lead to human error.

Among the solutions that have been proposed to mitigate those problems, one that has been widely adopted is the Neubauer chamber, also known as hemocytometer [8]. It consists of a thick glass microscope slide with a rectangular indentation that creates a chamber. This chamber is marked with a grid of perpendicular lines that define a number of regions with different known areas (see Fig. 1), and the depth of the chamber is also known. This makes it possible to count the number of objects in a certain region of interest, and from that calculate the overall concentration of objects.

The method presented in this paper goes a step further into the benefits provided by the geometrical grid of Neubauer chambers, by employing image processing techniques to fully automate the counting process. The main challenge tackled in this proposal is the correct delimitation of the regions of interest (ROI), from which the objects concentrations are estimated. Once the ROIs are defined, the actual counting may

Jayme Garcia Arnal Barbedo, Embrapa Agricultural Informatics, Campinas-SP, Brazil, E-mail: jayme.barbedo@embrapa.br

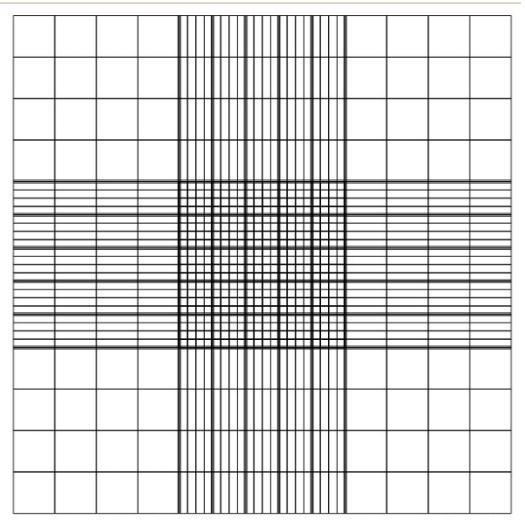

Fig. 1. The Neubauer chamber grid.

be performed, with little to no adaptation, using one of the many counting methods found in the literature, as illustrated in Section III.

\section{Neubauer Chamber Counting}

As it can be seen in Fig. 1, the Neubauer chamber has a grid of vertical and horizontal lines whose crossings generate a number of squares and rectangles that are used as reference for the counting process. Not all regions are used in the concentration estimations, and the regions to be considered depend on the size of the objects. Details about region selection can be found in [9].

Objects that are totally inside a given region are always counted. On the other hand, objects touching the borders may or may not be considered. There are no mandatory rules regarding those bordering objects, but the most widespread strategy is to count objects that touch the upper and left bounds, and disregard objects that touch the lower and right limits [9] (see Fig. 2). This information is important because the definition of the areas of interest by the algorithm must reflect exactly those rules, as will be seen next section.

\section{The Method}

The first step of the algorithm is to convert the image into a grayscale, if necessary. Fig. 3 shows an example of a Neubauer chamber containing fungi spores. Most spores are too small to be seen clearly in the figure, but they can be better visualized in other images presented later in the paper. Besides, at this point the most important image feature is the chamber's grid.

Next, the Otsu's thresholding [10] is applied and the holes in the resulting binary image are filled (Fig. 4). Otsu's thresholding was used here because it deals better with glaring effects that sometimes appear in the borders of the area of interest. The mask defined by the binary image is applied to the gray 


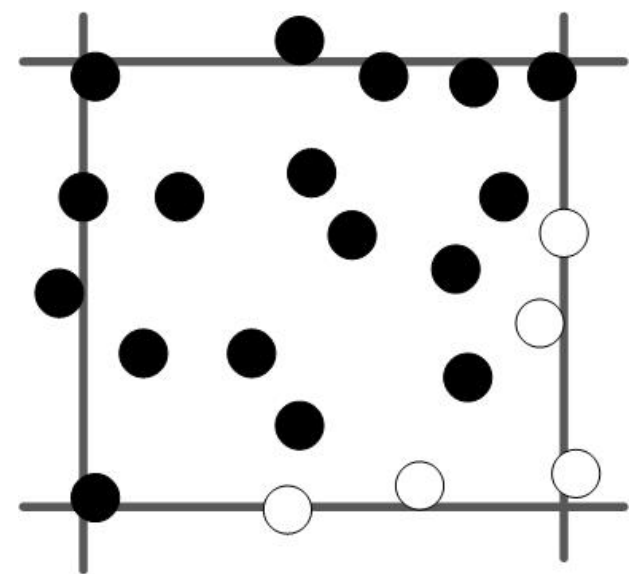

Fig. 2. Counting objects using the Neubauer chamber.

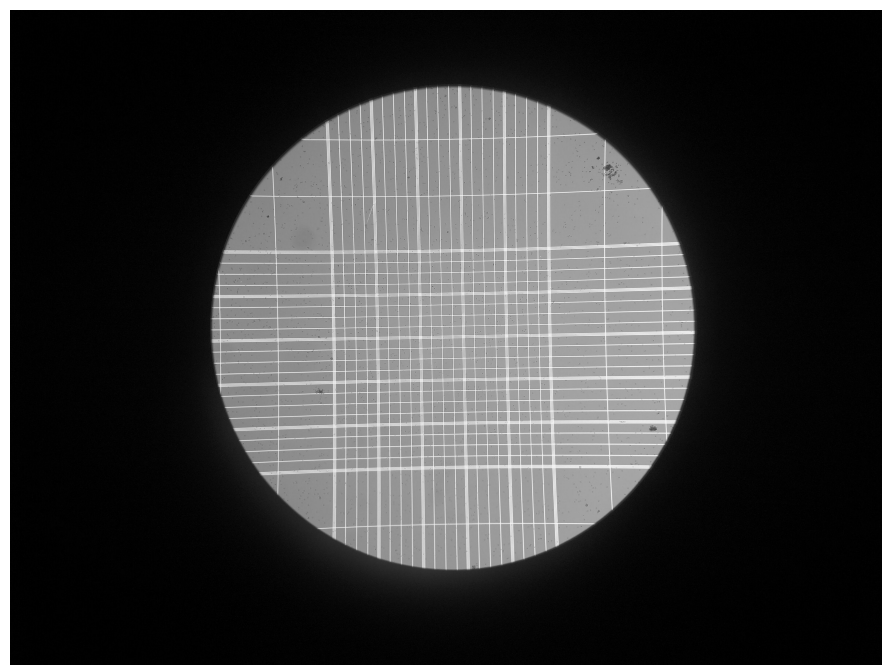

Fig. 3. Grayscale version of the Neubauer chamber image. This example will be used throughout the paper. Most spores are too small to be seen clearly here, but they can be better visualized in other images presented later in the paper.

image, which is cropped to include only the area of interest (Fig. 5).

The Sobel edge detection is then applied, as can be seen in Fig. 6. As a result, all edges from the chamber lines and objects are detected. At this point, the intention is to define the regions, and not in identifying the objects. Most objects are small and round, which is true not only for the example used in this paper, but also for most countings performed using the Neubauer chamber. On the other hand, the grid is composed by lines that are nearly straight - they are actually perfectly straight in the chamber but, due to the barrel lens distortion, they usually bend by a certain amount in the image. Inspired by those observations, two morphological openings are applied separately to the edge image:

- The first one uses a 1 by 10 pixels horizontal line as morphological structuring element (MSE) (for images with less than 4 Megapixels, a 1 by 6 pixels line is used instead). As a result, only elements strongly horizontal remain (Fig. 7). Although those values fit well most situations found in practice, it is important to emphasize that factors other than the size of

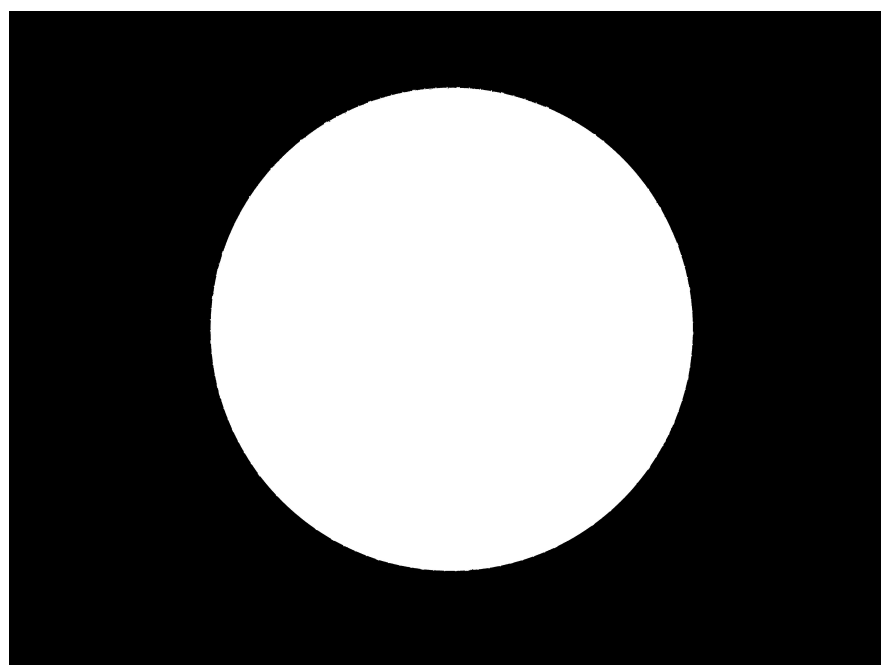

Fig. 4. Mask containing the area of interest.

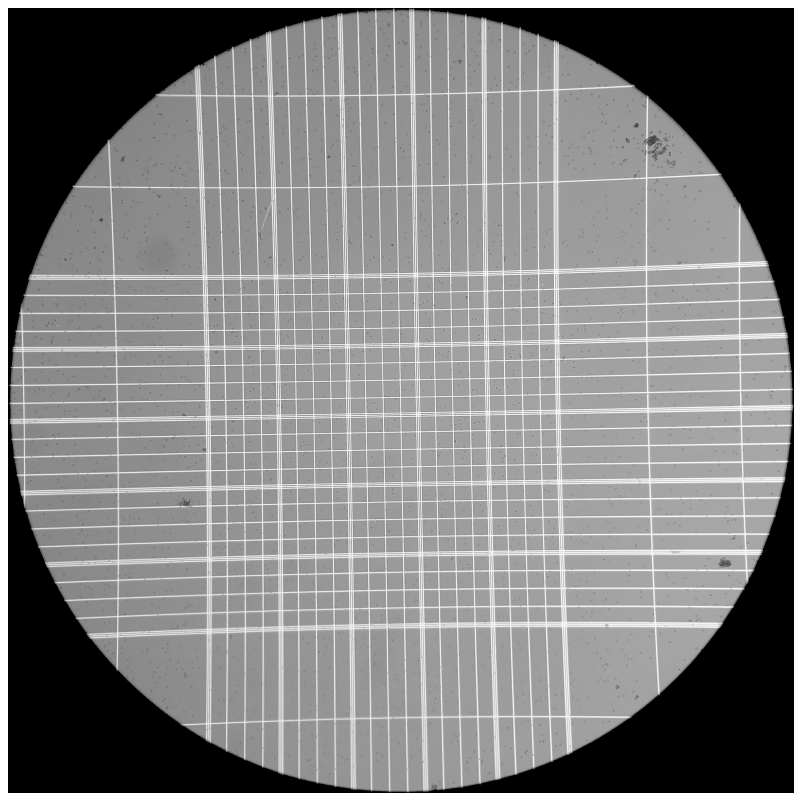

Fig. 5. Image cropped according to the mask.

the image, like zoom and sensor resolution, may influence the ideal length values, so those factors may sometimes need to be taken into account.

- The second one uses a 10 pixel vertical line as MSE. As a result, only elements strongly vertical remain (Fig. 8).

Finally, the two binary images are combined using the OR logical operator, resulting in an image containing only the chamber's grid (Fig. 9). It can be noticed in Fig. 9 that the edges present some flaws near the image extremities. This is due to a slight loss of sharpness, as the focus normally is adjusted having the center of the image as reference. This is usually not a big problem, as the center region is usually where the counting is performed. However, some of those flaws are corrected by the morphological closing of the image, which uses a square as MSE. The size of the square side is given by twice the number of megapixels of the original image (this value was determined empirically). As can be seen in Fig. 10, 


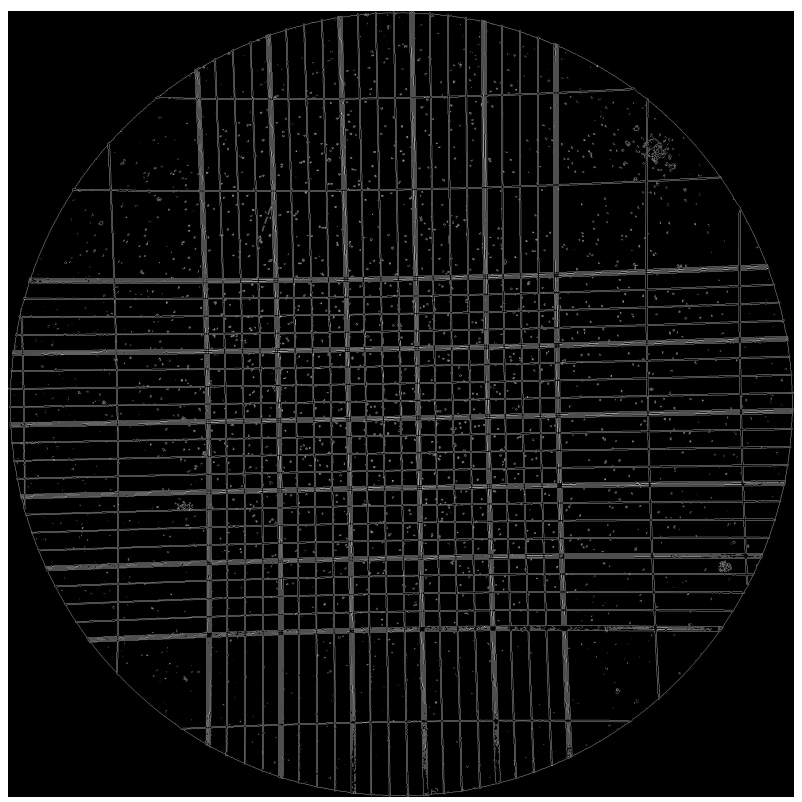

Fig. 6. Edges of the image using Sobel's approach.

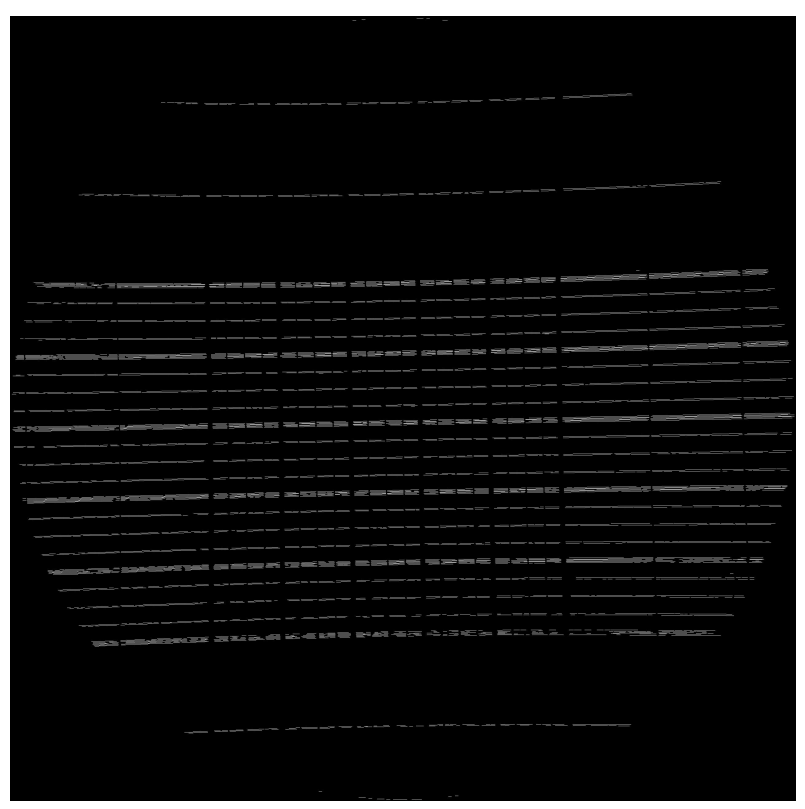

Fig. 7. Horizontal lines detection.

besides correcting some of the flaws, this closing also unites close lines into one single wider line, which is desirable when delimiting the regions, as will be seen later in this section.

At this point, there are still some line gaps that should be corrected in order to correctly identify the regions. In order to close those remaining gaps, the following rules are applied. For each row of the image, the algorithm identifies sequences of zeros that are bounded by sequences of ones whose lengths are at least half the length of the sequence of zeros. In this case, the zero-valued pixels are made equal to 1 . The same is carried out for the columns of the image. This effectively close gaps without changing the value of pixels not belonging to the lines. The result is shown in Fig. 11.

Next, the complement of the image is found (Fig. 12), and

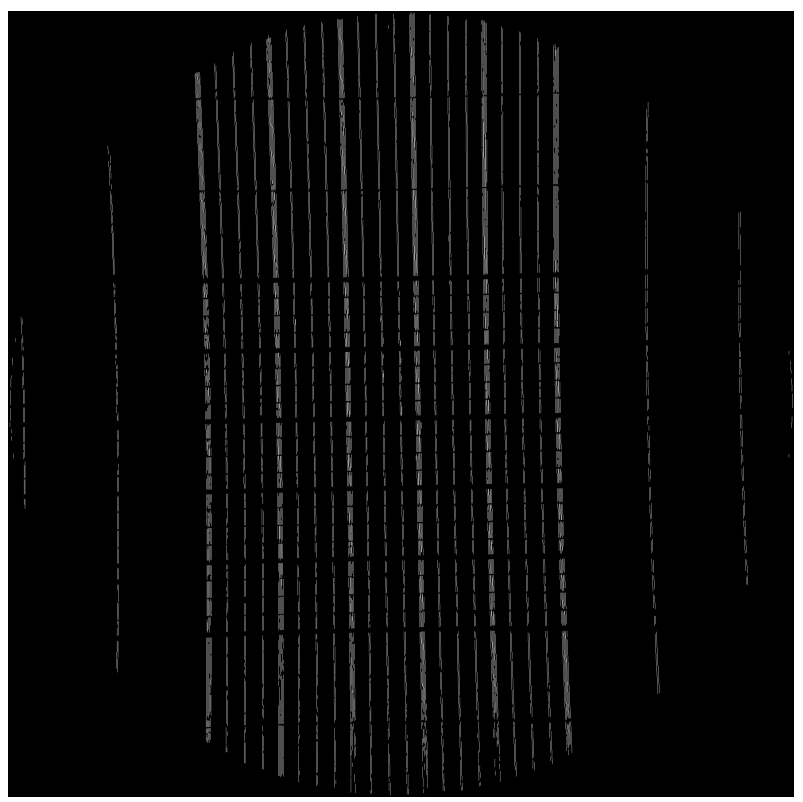

Fig. 8. Vertical lines detection.

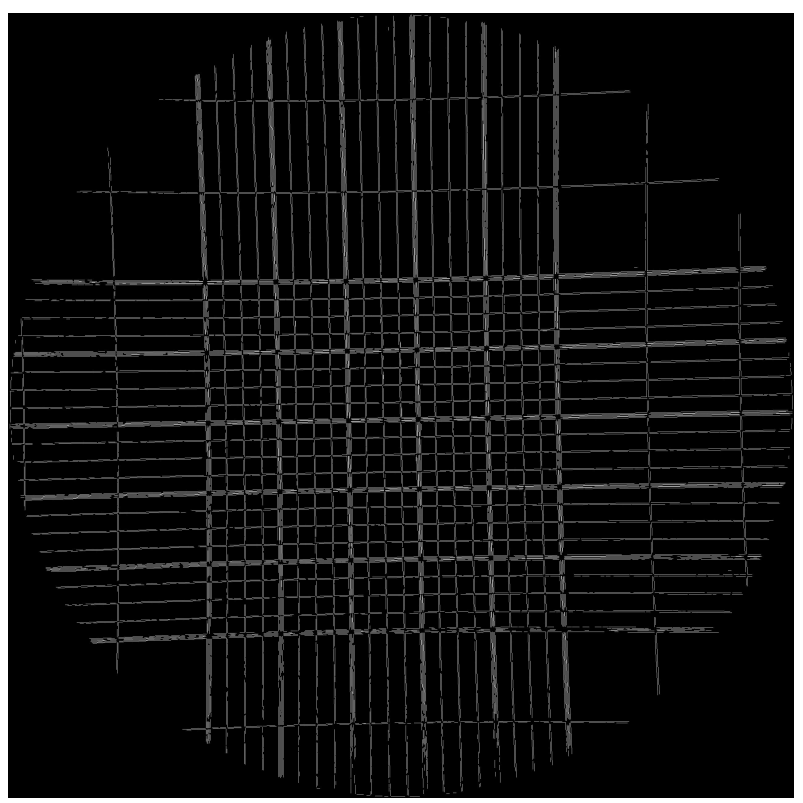

Fig. 9. Image after object edge removal.

the connected objects are identified and labeled. At this point, the regions are mostly identified. However, the lines of the grid are an important part of those regions, as objects touching those lines also need to be counted. Therefore, the regions occupied by the lines must be assigned to the correct regions according to the rules stated in Section 2. This is done as follows:

- First, serrated lines due to a tilt in the image or to the barrel lens effect are compensated, but only for the sides of the region that are to be incorporated to the region (upper and left, as stated in Section 2). An example of this compensation is shown in Fig. 13, where the pixels marked in red are made white, while the pixels inside the ellipse are kept black, as they should be assigned to the region below. 


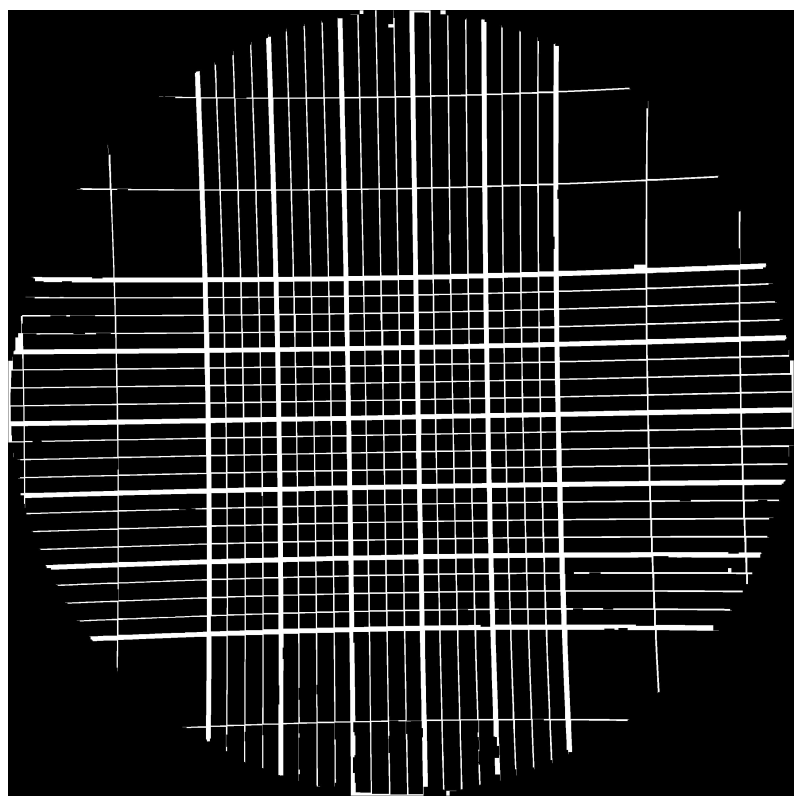

Fig. 10. Delimitation of the line regions.

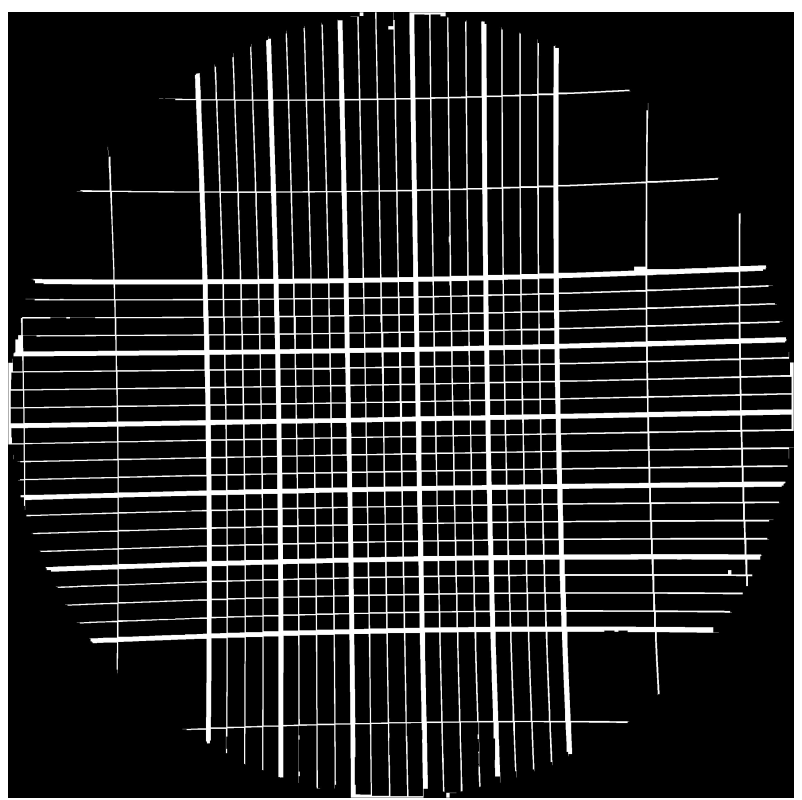

Fig. 11. Image after closing the gaps in the lines.

- In the following, black pixels that are located to the left and to the top of the current region are incorporated to that region. Here, some rules also apply. Fig. 14 illustrates those rules.

After the above rules are applied to all regions, every pixel in the image is assigned to a region. Next section presents some tests and remarks about the effectiveness of the proposed strategy.

\section{TESTS AND RESUlTS}

For the tests, four images of Neubauer chambers containing spores of the Clonostachys rosea fungus were captured. The images have 15 MPixels, and were originally captured using the RGB color representation.

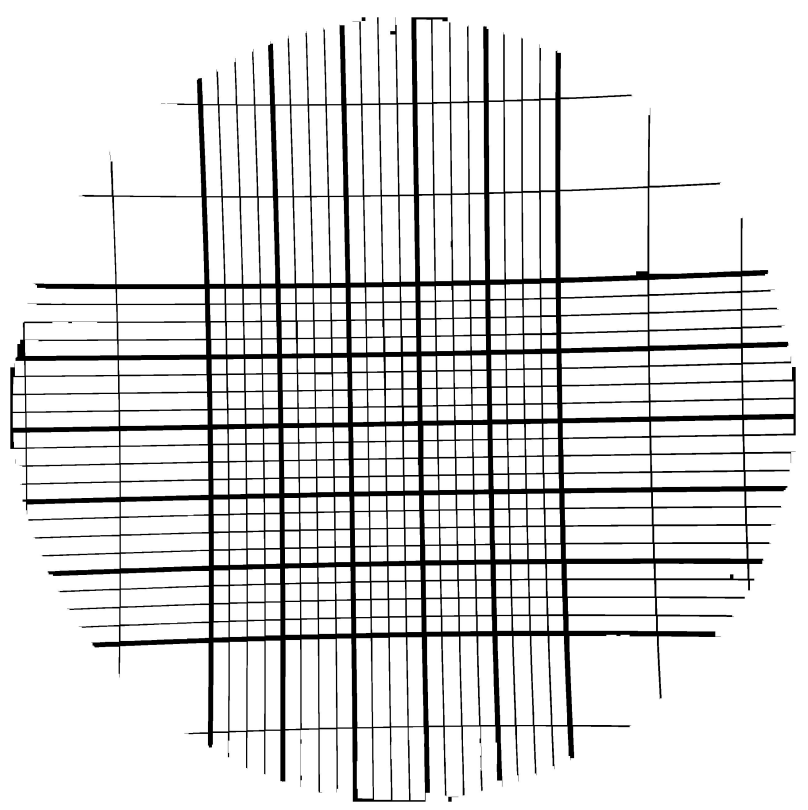

Fig. 12. Identification of the individual regions.

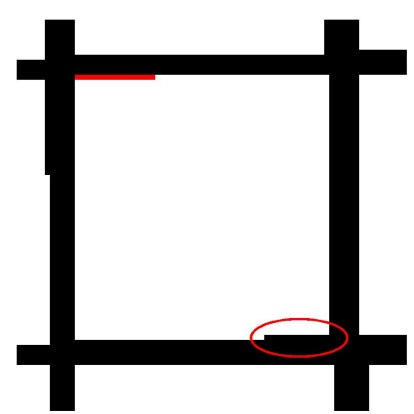

Fig. 13. Compensation to account for barrel lens effect.

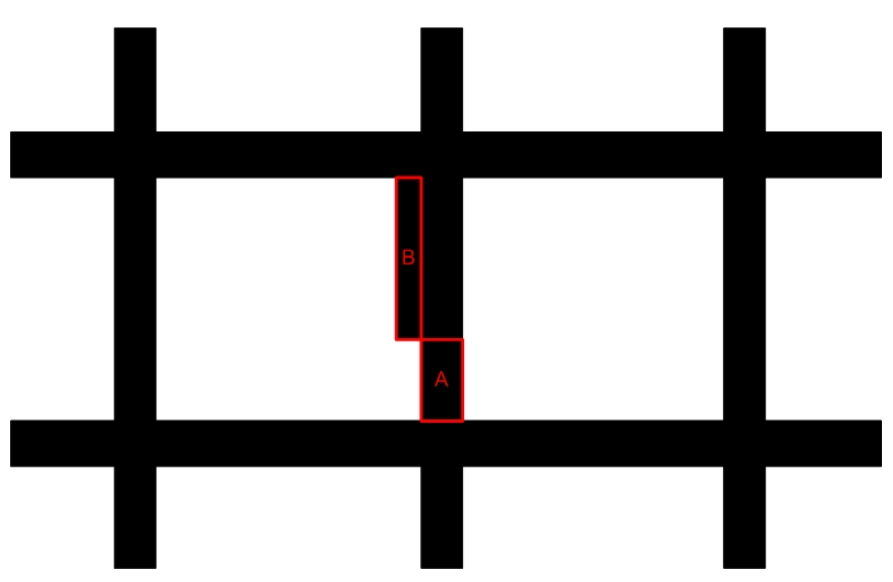

Fig. 14. Rules for assigning the line pixels to the correct regions. (A) Those pixels are assigned to the region on the right, according to the rules stated in Section II. (B) In this ambiguous situation, the pixels could be assigned to the region right next to them, as there are pixels from that region below them, or to the region on their right; in ambiguous situations like this, pixels are assigned to the region on the left (arbitrary rule). 
TABLE I

RESULTS.

\begin{tabular}{ccccccc}
\hline Resolution & $15 \mathrm{MP}$ & $10 \mathrm{MP}$ & $5 \mathrm{MP}$ & $2 \mathrm{MP}$ & $1 \mathrm{MP}$ & $0.5 \mathrm{MP}$ \\
\hline CAR & $97 \%$ & $96 \%$ & $95 \%$ & $90 \%$ & $82 \%$ & $64 \%$ \\
\hline
\end{tabular}

Overall, considering the rules presented in Section 2, a little more than $97 \%$ of the pixels were assigned to the correct region when compared to a manually labeled image. If the image is reduced by $20 \%$ by removing the border areas, the correct assignment rate is close to $99 \%$. Considering that the manual labeling is also subject to mistakes and ambiguity, this result means that the automatic labeling system proposed here is as effective as the manual one.

Those results were obtained using high resolution images. In order to investigate the influence of the resolution, the images were downsampled by successively increasing factors. Table I shows the correct assignment ratios (CAR) for some selected resolutions.

As can be seen in Table I, for resolutions above 5 MPixels the results are practically the same, since the small differences are mostly due to distortions caused by the downsampling process. On the other hand, lower resolutions cause the lines to be represented by very few pixels, making it very difficult for the algorithm to find their edges and to compensate for eventual gaps. Therefore, it is recommended that the images have at least 5 MPixels in order to avoid unreliable results.

Another important factor is the focus of the lens. As commented before, the results for the edges of the image are poorer due to a slight loss of focus. This indicates that a correct adjustment of the focus prior to the image capture is essential.

As stated before, the technique proposed here can be used in conjunction with almost any automatic object counting technique. In order to test the proposal up to the point of the final estimate for the number of objects, the counting method proposed in [5] was adopted. The object detection is shown in Fig. 15 - only a small part of the image is shown so the small objects could be visible. As can be seen, only two objects that touch the lines were not identified, but this mistake came from the counting algorithm, and not from the region delimitation. The complete tool combining both the region delimitation and the object counting was implemented with an interface that allows the user to visualize the counting estimates for individual regions and also for sets of regions. The interface is not shown here due to space limitations.

Finally, because the algorithm is based mostly on simple mathematical morphology and straightforward heuristic rules, it is computationally inexpensive, taking no more than a few seconds to process a 15-MPixel image even in situations with low computational resources.

\section{Conclusions}

This paper presented a method to identify and finely mark out the limits of each region of interest in a Neubauer chamber. The identification of the central parts of the regions is relatively straightforward, but assigning the line areas to the correct regions demanded a more sophisticated approach.

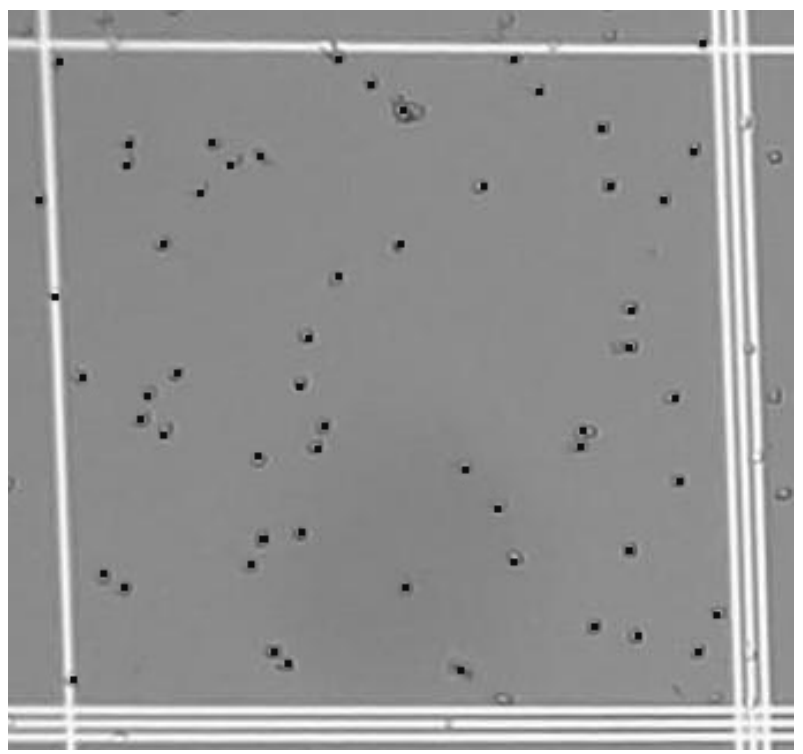

Fig. 15. Example of object identification.

The resulting algorithm is capable of correctly assigning more than $97 \%$ of the pixels. In order for the method to work properly, it is recommended that the images have more than 5 MPixels, and the focus should be carefully tuned to guarantee the sharpness of the image. Future work will concentrate on the improvement of the integration between the region delimitation and counting algorithms, in such a way the results from one module can be fed back to the other one so the overall results can be refined.

\section{REFERENCES}

[1] G. Cordiki, R. Diaz-Uribe, J. L. Folch-Mallol, and J. Nieto-Sotelo, "COVASIAM: an image analysis method that allows detection of confluent microbial colonies and colonies of various sizes for automated counting," Applied and Environmental Microbiology, vol. 64, pp. 1400-1404, 1998.

[2] J. Marotz, C. Lübbert, and W. Eisenbeiß, "Effective object recognition for automated counting of colonies in Petri dishes (automated colony counting)," Computer Methods and Programs in Biomedicine, vol. 66, pp. 183-198, 2001.

[3] J. Selinummi, J. Seppälä, O. Yli-Harja, and J. Puhakka, "Software for quantification of labeled bacteria from digital microscope images by automated image analysis," BioTechniques, vol. 39, pp. 859-862, 2005.

[4] J. G. A. Barbedo, "Method for counting microorganisms and colonies in microscopic images," in Proc. International Conference on Computational Science and Its Applications, 2012, pp. 83-87.

[5] — , "Unified framework for counting agriculture-related objects in digital images," in Proc. Workshop on Industry Applications (WGARI) in SIBGRAPI 2012 (XXV Conference on Graphics, Patterns and Images), 2012, pp. 42-48.

[6] _ "Method for automatic counting root nodules using digital images," in Proc. International Conference on Computational Science and Its Applications, 2012, pp. 159-161.

[7] C. R. M. Mauricio, F. K. Schneider, and L. Santos, "Image-based red cell counting for wild animals blood," in Proc. Int. Conf. IEEE Eng. in Medicine and Biology Society, 2010, pp. 438-441.

[8] A. C. Lucarini, L. A. Silva, and R. A. C. Bianchi, "Um sistema para a contagem semi-automática de microorganismos," Pesquisa e Tecnologia FEI, vol. 26, pp. 36-40, 2004.

[9] O. Bastidas, "Cell counting with neubauer chamber," Celeromics, Tech. Rep., 2013.

[10] N. Otsu, "A threshold selection method from gray-level histograms," IEEE Transactions on Systems, Man and Cybernetics, vol. 9, no. 1, pp. 62-66, 1979. 